\title{
Validation of FENDL-2.1 nuclear data library for use in ITER nuclear analysis
}

\author{
P. Batistoni ${ }^{1}$, L. Petrizzi ${ }^{1}$, U. Fisher ${ }^{2}$, A. Serikov ${ }^{2}$, K. Seidel ${ }^{3}$, R. Villari ${ }^{1}$, and L. Auditore ${ }^{1}$ \\ 1 Associazione EURATOM-ENEA sulla Fusione C. R. Frascati, Via Enrico Fermi, 45, 00044 Frascati (Rome), Italy \\ 2 Association FZK-Euratom, Forschungszentrum Karlsruhe, Hermann-von-Helmholtz-Platz 1, 76344 Eggenstein-Leopoldshafen, Germany \\ 3 TU Dresden, Institut für Kern- und Teilchenphysik, 01062 Dresden, Germany
}

\begin{abstract}
Nuclear design for ITER having an impact on components, equipment, and materials, must conform to established requirements, and must be fully traceable to valid data, and capable of withstanding detailed technical reviews. According to the Management and Quality Program that is being established in the ITER project, nuclear data used for neutronic calculations must be verified and validated prior to use in ITER design. Extensive work of validation and verification FENDL-1 and FENDL-2.0 libraries for neutronic calculations was performed during the ITER R\&D activities through ad hoc benchmark experiments performed on mock-ups of ITER components at $14 \mathrm{MeV}$ neutron generators. In particular, this paper presents the validation of the latest version FENDL-2.1 using the existing benchmark experiments carried out at the FNG $14 \mathrm{MeV}$ neutron generator at ENEA Frascati.
\end{abstract}

\section{Introduction}

The ITER Project is realizing the first magnetic fusion facility that will produce significant fusion power, and that requires nuclear licensing. According to French regulation the ITER device will be classified as a nuclear installation called "Installation Nucléaire de Base" (INB) due to the expected tritium inventory and the expected waste generation during the lifetime of ITER. The aim of ITER is to construct and operate such first-of-the-type nuclear fusion installation satisfying the demands and interests of the user community and of funding partners.

All activities, including nuclear design with results for facilities, structures, systems, components, equipment, and materials, must conform to established requirements, and must be fully traceable to valid data, and capable of withstanding detailed technical reviews. According to the Management and Quality Program that is being established in the ITER project, nuclear data used for neutronic calculations must be verified and validated [1].

In order to provide adequate nuclear data for ITER design, the international Fusion Evaluated Nuclear ata Library (FENDL) was established in 1987 under the IAEA coordination in cooperation with national nuclear reaction data centers and research groups. This library is a comprehensive collection of high-quality nuclear data, selected from the various existing national data libraries, tailored to the requirements of fusion development projects. The first version of the library, FENDL-1, was officially released early in 1995 followed by FENDL-2.0 which was finalized for general distribution in January 1999, and recently updated in version FENDL-2.1 [2]. This last revised and improved version of FENDL is the reference nuclear data library for ITER nuclear design.

Extensive work of validation and verification of FENDL1/2.0 libraries for neutronic calculations was performed during the R\&D activities for ITER design through ad hoc benchmark experiments performed on mock-ups of components at $14 \mathrm{MeV}$ neutron generators. In particular, this paper presents

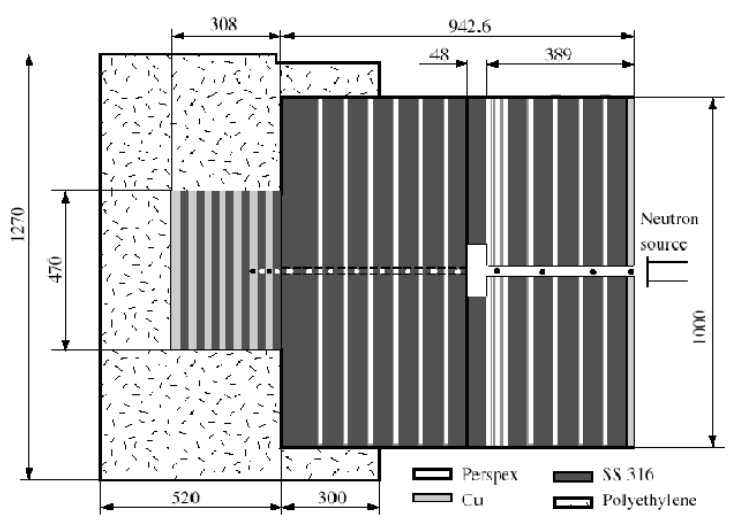

Fig. 1. Layout of the Streaming Experiment (the Bulk Shield Experiment used the same block without channel and cavity).

the validation of the latest version FENDL-2.1 using the existing benchmark experiments carried out at the FNG $14 \mathrm{MeV}$ neutron generator at ENEA Frascati. They investigate a wide spectrum of nuclear relevant issues for ITER, including those related to shielding blankets (with and without streaming paths), breeding blanket, vessel, magnets, and divertor. A comparison with previous FENDL version and with the European EFF-3.1 and JEFF-3.1 libraries [3] is discussed as well.

\section{Benchmark experiments}

The first integral experiment considered here is the Bulk Shield Experiment, consisting in a mockup of the ITER shielding blanket/vacuum vessel (simulated by alternating layers of stainless steel, AISI-316-type, (SS316) and Perspex material to simulate water) followed by the superconducting magnet region (simulated by alternating layers of SS316 and copper) [4]. The neutron and gamma ray flux were measured as a function of depth in the shielding blanket/vessel, and the nuclear heating was measured all along the mock-up depth 


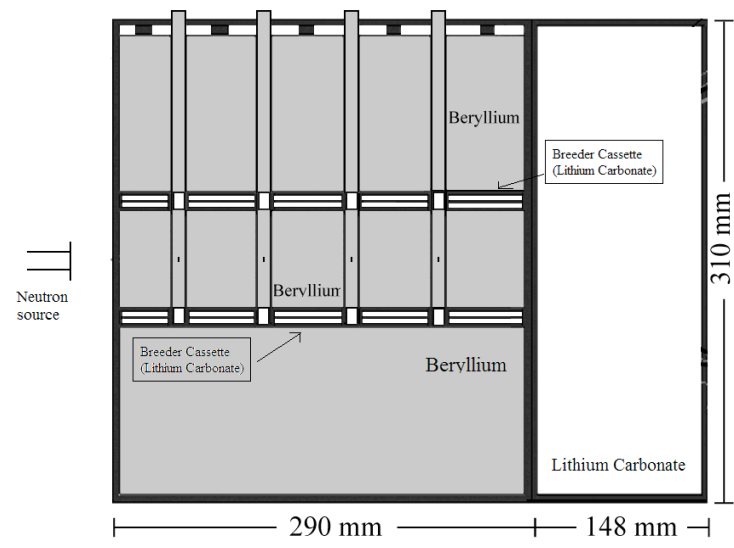

Fig. 2. Layout of the TBM-HCPB Experiment.

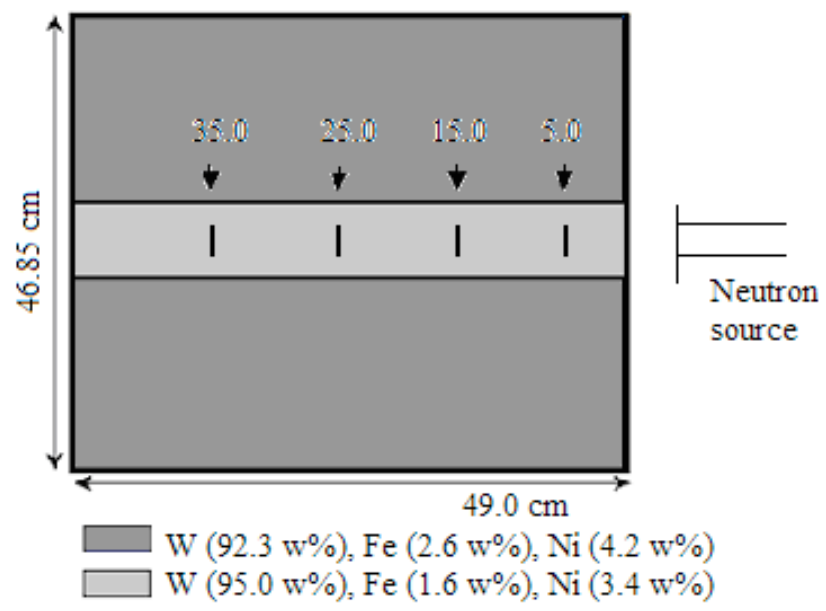

Fig. 3. Layout of Tungsten Experiment with detector positions.

and in the magnet region. The second integral experiment, the Streaming Experiment, consisted in a variation of the previous one, where a circular central channel and a rectangular cavity were introduced in the shielding blanket/vessel block to simulate steaming paths between ITER blanket modules [5]. The arrangement of this experiment is shown in figure 1. Neutron flux was measured by activation technique along the central channel and inside the cavity at locations out-of-site of the point source and thus measure the degree of streaming through the central channel. The third experiment is the TBM-HCPB Experiment [6], on a mock up the Test Blanket Module - Helium Cooled Pebble Bed concept, to be tested in ITER (fig. 2). In this experiment, the neutron flux was measured as a function of depth in the beryllium block in the module, the neutron and gamma ray flux spectra were measured behind the module, and the tritium production rate was measured in the Li composite in the breeder cassettes. Finally, the Tungsten Experiment [7] is considered, as tungsten is used in ITER divertor as armor material. It consisted in a rectangular block whose dimensions are shown in figure 3 . The neutron and gamma flux, as well as nuclear heating, were measured as a function of depth in the block.

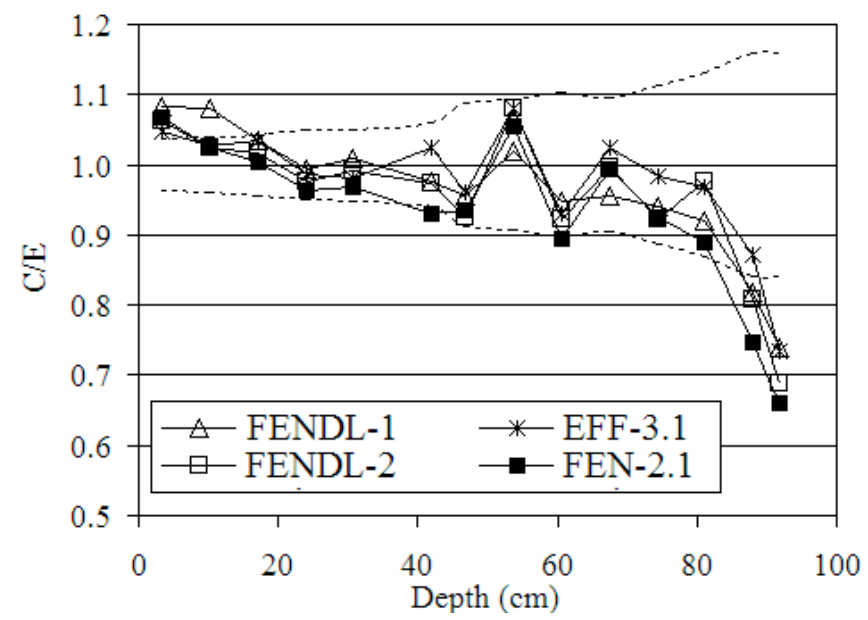

Fig. 4. Calculated (C) over measured (E) fast neutron flux $(\mathrm{E}>\sim 1 \mathrm{MeV})$ in the Bulk Shield Experiment. Measured flux is obtained by activation foils using the Ni-58(n,p) reaction. Dotted lines represent the total uncertainties (experiment + calculation) in the comparison.

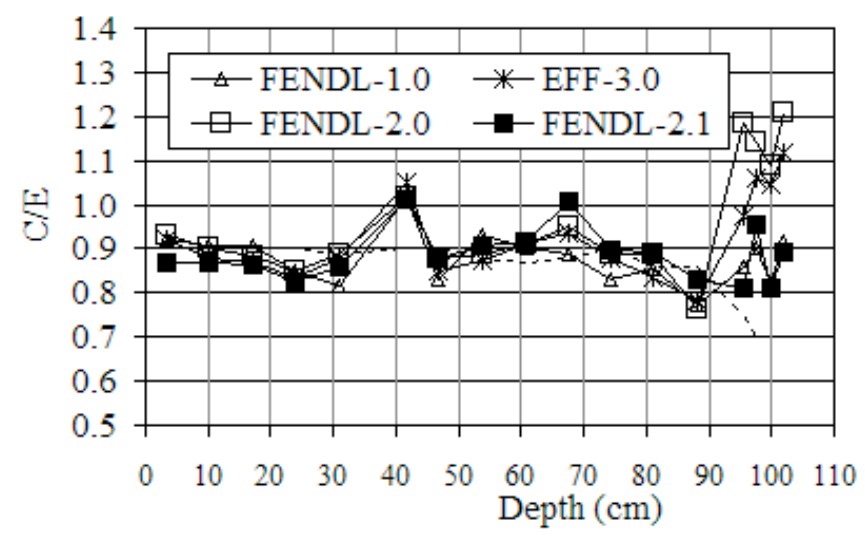

Fig. 5. Analysis of nuclear heating in the Bulk Shield Experiment, as measured by thermo luminescent dose meters (TLD-300).

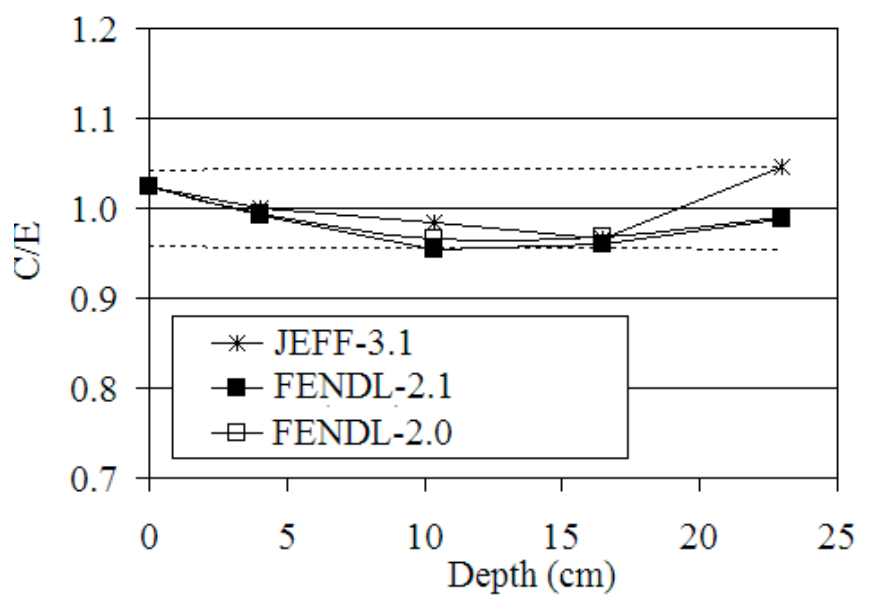

Fig. 6. Analysis of fast neutron flux $(\mathrm{E}>\sim 10 \mathrm{MeV})$ in the $T B M$ $H C P B$ Experiment, as measured by the $\mathrm{Nb}-93(\mathrm{n}, 2 \mathrm{n})$ reaction. 
Table 1. Origin of data in FENDL-1/2.0/ 2.1 for main materials present in ITER experiments carried out at FNG.

\begin{tabular}{llll}
\hline Materials & FENDL-1.0 & FENDL-2.0 & FENDL-2.1 \\
\hline Fe-56 & E/B-VI & EFF-3 & EFF-3 \\
Fe-54,57-58 & E/B-VI & E/B-VI & E/B-VI \\
\hline Cr-52 & E/B-VI & E/B-VI & E/B-VI \\
\hline Ni-58, 60 & E/B-VI & E/B-VI & EFF-3 \\
& & E/B-VI & EFF-3 \\
\hline W-182, & \multirow{2}{*}{ E/B-VI } & JENDL-FF & E/B-VI \\
183-4-6 & (W-nat) & \\
\hline Li-6 & E/B-VI & E/B-VI & E/B-VI \\
Li-7 & E/B-VI & E/B-VI & E/B-VI \\
\hline Be-9 & E/B-VI (C-nat) & JENDL-FF & JENDL-FF \\
\hline $\mathrm{C}-12$ & E/B-VI (C-nat) & JENDL-FF & JENDL-FF \\
\hline $\mathrm{O}-16$ & E/B-VI & JENDL-FF & E/B-VI \\
\hline
\end{tabular}

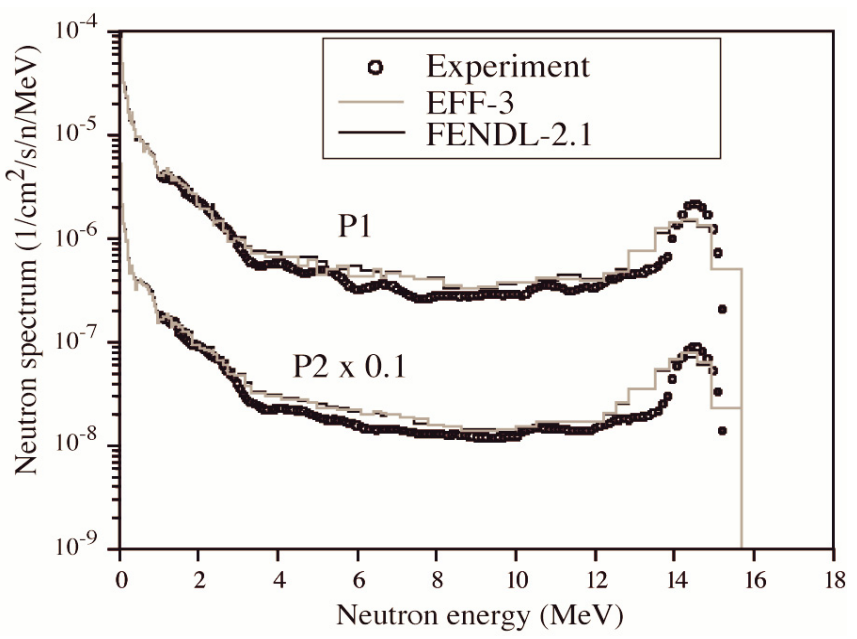

Fig. 7. Calculated (C) and measured (E) fast neutron flux in the $T B M$ $H C P B$ Experiment. ( $\mathrm{P} 1$ is the position inside the mock-up, $\mathrm{P} 2$ is behind the mock-up).

\section{Analysis}

All experiments have been analysed with FENDL-2.1and using the MCNP-4c/5 code. In all cases, very detailed geometrical models of the experimental set ups have been used, including the detectors employed in the measurements, the neutron generator and the bunker hall. The results obtained with FENDL-2.1 have been compared with those obtained with FENDL-2.0 and JEFF-3.1 (EFF-3). In table 3, the original sources of data in the three FENDL releases are given for the main materials present in the experiments.

\subsection{Bulk shield and streaming experiments}

In both experiments the fast neutron fluxes are underestimated by FENDL-1.0 (main materials in stainless steel from ENDF/B-VI), by FENDL-2.0 and also by FENDL-2.1 (main materials in stainless steel from ENDF/BVI, Fe-56 and Ni58,60 from EFF-3) up to about $10 \%$ at $40 \mathrm{~cm}$ depth, and $25-30 \%$ at about $1 \mathrm{~m}$ depth in the block (see fig. 4). The gamma ray flux is also underestimated by $\sim 20 \%$ at $1 \mathrm{~m}$ depth.

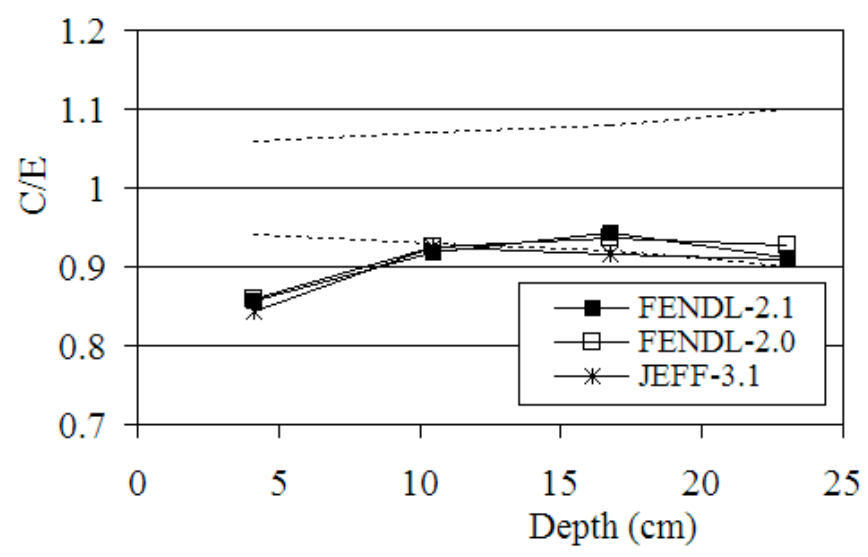

Fig. 8. Calculated $(\mathrm{C})$ over measured (E) tritium production rates as a function of depth in the lower cassette in the $T B M-H C P B$ Experiment. Dotted lines represent the total uncertainties (experiment + calculation) in the comparison.

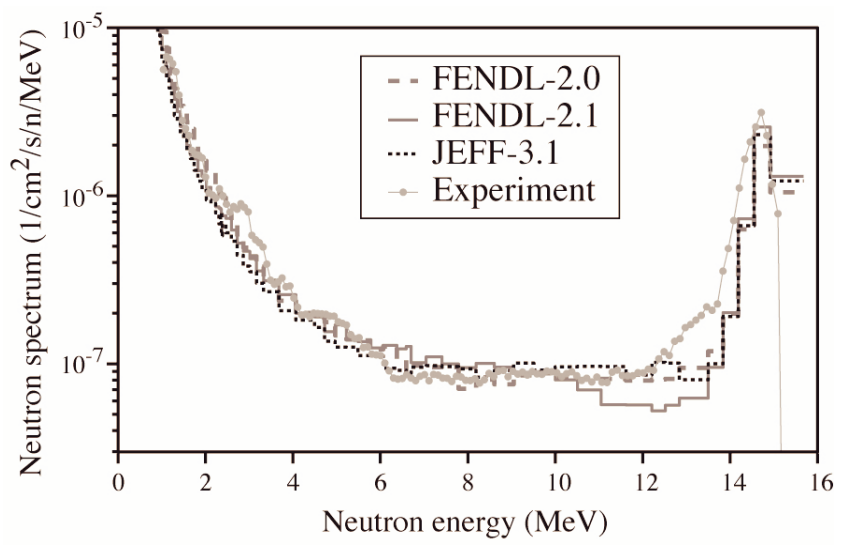

Fig. 9. Calculated (C) and measured (E) fast neutron flux in the Tungsten Experiment at $25 \mathrm{~cm}$ depth (pos. 3).

A better agreement is found for the thermal neutron flux (within $\pm 10 \%$ ). Nuclear heating (fig. 5): as a matter of fact, no significant differences are found between the three FENDL versions. From these results it is concluded that the neutron and gamma ray fluxes are predicted in stainless steel/water shield assemblies by FENDL-2.1 within +/- 30\% uncertainty at $1 \mathrm{~m}$ depth.

\subsection{TBM-HCPB experiments}

As a general result, the neutron flux in the Be layer is well predicted by FENDL-2.0/2.1 and by JEFF-3.1 within the total combined uncertainties $(\sim \pm 5 \%)$ up to about $24 \mathrm{~cm}$ depth. The ratio of calculated $(\mathrm{C})$ over measured $(\mathrm{E})$ fast neutron flux is shown in figure 6. No significant differences between FENDL2.0/2.1 and JEFF-3 are observed. The fast neutron flux (E > $1 \mathrm{MeV}$ ) was found to be slightly overestimated by about $10 \%$ behind the mock-up (P2 in fig. 7). This indicates that shielding calculations for the HCPB blanket are conservative. The $\gamma$-ray flux is underestimated by all libraries by about $10 \%$ at the back of the mock-up. The slow neutron flux investigated by timeof-arrival spectroscopy is underestimated in the mock-up by 


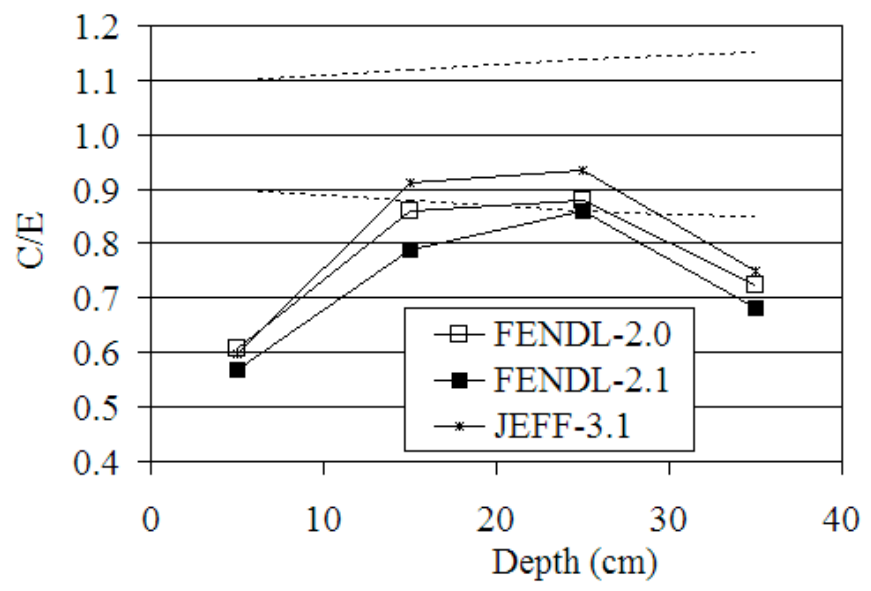

Fig. 10. Calculated (C) over measured (E) nuclear heating in tungsten in the Tungsten Experiment. Dotted lines represent the total uncertainties (experiment + calculation) in the comparison.

about $20 \%$. Consistently, a slight underestimation is found in the calculation of the tritium production in the breeder material in the cassettes, the underestimation being smaller at deeper positions. $\mathrm{C} / \mathrm{E}$ average values for the tritium production rate range from 0.86 (front position) to 0.92 at larger depths (see fig. 8). Also in this case, no significant differences between FENDL-2.0/2.1 and JEFF-3 are observed.

\subsection{Tungsten experiment}

In the case of tungsten, FENDL-2.1 performs better than FENDL-2.0. In fact, an improvement is observed in the prediction of the fast neutron flux (fig. 9). The thermal neutron flux is well predicted by all libraries. The photon flux is overestimated at all depths, while the analysis of the nuclear heating, which is dominated by the neutron contribution, shows that it is underestimated by all libraries, especially in the front position (fig. 10).

\section{Conclusions}

FENDL-2.1, the reference nuclear data library for ITER design, has been validated using the existing benchmark experiments carried out at the FNG $14 \mathrm{MeV}$ neutron generator at ENEA Frascati. These experiments investigate a wide spectrum of nuclear relevant issues for ITER, including those related to shielding blankets (with and without streaming paths), breeding blanket, vessel, magnets, and divertor. A comparison with previous FENDL version and with the European EFF-3.1 and JEFF-3.1 libraries [3] is discussed as well.

From these results it is concluded that the neutron and gamma ray fluxes are predicted in stainless steel/water shield assemblies by FENDL-2.1 within $+/-30 \%$ uncertainty at $1 \mathrm{~m}$ depth. A slight underestimation is found in the calculation of the tritium production rate in the Test Blanket Module mock-up simulating the breeder blanket, HCPB type, the underestimation by $10-14 \%$ being comparable with the total combined uncertainty of the experiment.

Finally, the thermal neutron flux in tungsten is well predicted by all libraries, while the analysis of the nuclear heating shows that it is underestimated by especially in the front position.

As a general result, it is concluded that FENDL-2.1 data library suitable for fusion design applications calculations. However, it could be further improved to reduce the uncertainty margins in the ITER design.

This work, supported by the Euratom Communities under the contract of Association between EURATOM-ENEA, was carried out within the framework of the European Fusion Development Agreement. The views and opinions expressed herein do not necessarily reflect those of the European Commission.

\section{References}

1. P. Batistoni, Ken Sowder, Quality Assurance in Neutronic Analyses, ITER Report, May 2006, https://users.iter.org/ users/idm?document_id=ITER_D_23H9A4.

2. D.L. Aldama, A. Trkov, FENDL-2.1: Update of an evaluated nuclear data library for fusion applications, IAEA Report INDC(NDS)-467, December 2004.

3. A.J. Koning et al., The JEFF evaluated nuclear data project (this conference).

4. P. Batistoni, M. Angelone, U. Fischer, H. Freiesleben, W. Hansen, M. Pillon, L. Petrizzi et al., Neutronics experiment on a mock-up of the ITER shielding blanket at the Frascati Neutron Generator, Fus. Eng. Design 47, 25 (1999).

5. M. Angelone, P. Batistoni, L. Petrizzi, M. Pillon, Neutron streaming Experiment at FNG: results and analysis, Fus. Eng. Des. 51-52, 653 (2000).

6. P. Batistoni, M. Angelone, L. Bettinali, P. Carconi et al., Neutronics experiment on a HCPB breeder blanket mock-up (accepted for publication in Fus. Eng. Des.).

7. P. Batistoni et al., Neutronics Benchmark Experiment on Tungsten, J. Nucl. Mater. 329-333, 683 (2004). 